\title{
An Enhanced Piezoelectric Vibration Energy Harvesting System with Macro Fiber Composite
}

\author{
Shuwen Zhang, ${ }^{1}$ Bo Yan, ${ }^{2}$ Yajun Luo, ${ }^{1}$ Weikai Miao, ${ }^{1}$ and Minglong Xu${ }^{1}$ \\ ${ }^{1}$ State Key Laboratory for Strength and Vibration of Mechanical Structures, School of Aerospace, Xian Jiaotong University, \\ Xianning West Road, No. 28, Xian 710049, China \\ ${ }^{2}$ Technology and Engineering Center for Space Utilization, Chinese Academy of Sciences, Dengzhuang South Road, No. 9, \\ Beijing 100094, China
}

Correspondence should be addressed to Minglong Xu; mlxu@mail.xjtu.edu.cn

Received 15 April 2015; Revised 7 July 2015; Accepted 12 July 2015

Academic Editor: Carlo Trigona

Copyright (C) 2015 Shuwen Zhang et al. This is an open access article distributed under the Creative Commons Attribution License, which permits unrestricted use, distribution, and reproduction in any medium, provided the original work is properly cited.

Self-power supply is a promising project in various applied conditions. Among this research area, piezoelectric material-based energy harvesting $(\mathrm{EH})$ method has been researched in recent years due to its advantages. With the limitation of energy form acceptance range of $\mathrm{EH}$ circuit system, a sum of energy is not accessible to be obtained. To enlarge the EH quantity from the vibration, an enhanced piezoelectric vibration $\mathrm{EH}$ structure with piezoelectric film is developed in this work. Piezoelectric-based energy harvesting mechanism is primarily proposed in this work. The special-designed electric circuit for EH from macro fiber composite (MFC) is proposed and then analyzed. When the structure vibrates in its modes of frequencies, the experiments are developed to measure the EH effect. The energy harvested from the vibrating structure is analyzed and the enhanced effect is presented. The results indicate that, with the enhanced $\mathrm{EH}$ structure in this work, vibration energy from structure is obtained in a larger range, and the general EH quantity is enlarged.

\section{Introduction}

Vibration on structures occasionally plays a negative role because of its disadvantages. As a solution, vibration control has been used to overcome the disadvantages [1,2]. Generally, active control, containing sensing, signal processing, and actuating module [2-5], is a major vibration control method. All of the above-mentioned modules in the vibration control system require electric power as necessity. In some applied conditions, power supply tends to be costly and hard to achieve $[6,7]$. Hence, self-power supply becomes an ideal solution. Recently, vibration energy harvesting technology, both acting as the function of energy harvesting (EH) and decreasing the vibration amplitude, has attracted research attention. With electromechanical EH methods like voice coil, smart materials, and other methods, energy is captured and then supplied to load such as wireless sensors and microelectromechanical systems (MEMS) [5, 8-10]. The captured electric energy is adjusted and further processed for load before use [11].
Among the various EH methods, piezoelectric energy vibration harvesting is widely studied. Compared with other methods, piezoelectric material occupies less space. Piezoelectric materials are widely used to harvest the mechanical energy for the long term and low power electric loads [12-14]. As the energy quantity requirement is as small as $\mathrm{mW}$ magnitude or even smaller, the piezoelectric materials are seen as ideal harvesters. Wideband energy harvesting technologies are presented to increase the energy obtaining efficiency [15-18]. With the wider acceptance range (vibration frequency and amplitude), the energy harvesting quantity is enlarged, and the EH ability is increased. Besides linear system, the nonlinear vibration based $\mathrm{EH}$ is analyzed and the effects of EH are also presented [11, 19-21]. Besides, wireless systems offer great flexibility, increased reliability, and reduced costs compared with a wired infrastructure. The majority of sensor nodes are reliant on battery, which will require periodical replacement, and are therefore not consistent with the concept of a permanent fully embedded system. A vibration energy harvesting based wireless power 
TABLE 1: Parameters of MFC and vibrating structure.

\begin{tabular}{|c|c|c|c|c|c|c|}
\hline Item & Type & Size $(\mathrm{mm})$ & Max strain & $C_{\mathrm{MFC}}$ & First mode & Second mode \\
\hline MFC & M2814 & $28 \times 14 \times 0.3$ & $1550 \mathrm{ppm}$ & $0.61 \mathrm{nF}$ & - & - \\
\hline Beam & $45 \#$ steel & $370 \times 20 \times 1.39$ & - & - & $8.42 \mathrm{~Hz}$ & $52.76 \mathrm{~Hz}$ \\
\hline MFC-beam & - & - & - & - & $8.30 \mathrm{~Hz}$ & $51.99 \mathrm{~Hz}$ \\
\hline
\end{tabular}

autonomous supply system with piezoelectric material is presented to solve this problem [22].

In this work, the piezoelectric material macro fiber composite (MFC) is used to harvest vibration energy. To enlarge the $\mathrm{EH}$ ability, an enhanced electric circuit is presented after MFC to adjust the captured AC electric energy. The experimental results indicate that with the enhanced electric circuit design the vibration energy self-supply ratio (VESR) is increased.

The rest of the paper is organized as follows. The energy harvesting mechanism is analyzed in Section 2. The experiment procedures are presented in Section 3. The energy harvesting ability is analyzed and discussed in Section 4. The conclusion is finally given in Section 5.

\section{Energy Harvesting Mechanism}

The electromechanical properties of the MFC-based energy harvesting system are analyzed in this section. The electromechanical effect of EH system is firstly presented, and then the captured energy is predicted in various conditions. The enhanced electric circuit is discussed and designed to enlarge the $\mathrm{EH}$ range. Figure 1 is the block diagram of active vibration control system with energy harvesting system in this work, while the bold line-connected parts are the focuses presented in this work.

\subsection{Electromechanical Properties of MFC-Cantilever Beam} Structure. The electromechanical properties on this experiment are the premise of energy harvesting in this work. A cantilever beam with MFC is presented for this experiment.

According to the elastic assumption and piezoelectric theory, the electromechanical properties of MFC are simplified as

$$
\left[\begin{array}{c}
\varepsilon_{x} \\
\varepsilon_{y} \\
\varepsilon_{z}
\end{array}\right]=\left[\begin{array}{lll}
S_{11}^{E} & S_{12}^{E} & S_{13}^{E} \\
S_{12}^{E} & S_{11}^{E} & S_{13}^{E} \\
S_{13}^{E} & S_{13}^{E} & S_{33}^{E}
\end{array}\right]\left[\begin{array}{c}
\sigma_{x} \\
\sigma_{y} \\
\sigma_{z}
\end{array}\right]+\left[\begin{array}{l}
d_{31} \\
d_{31} \\
d_{33}
\end{array}\right] E_{z}
$$

where $\varepsilon$ is the strain, $S^{E}$ is the elastic flexibility of MFC, $\sigma$ is the stress, $d$ is the piezoelectric strain constant, and $E_{z}$ is the electric field intensity, respectively.

The electric displacement of the MFC is simplified as

$$
D_{z}=\left[\begin{array}{lll}
d_{31} & d_{31} & d_{33}
\end{array}\right]\left[\begin{array}{c}
\sigma_{x} \\
\sigma_{y} \\
\sigma_{z}
\end{array}\right]+\varepsilon_{33}^{\sigma} E_{z}
$$

According to the definition of electric displacement, the $D_{z}$ can also be described as

$$
D_{z}=\frac{Q}{A}=\frac{C_{\mathrm{MFC}} U}{A_{e}}=\frac{C_{\mathrm{MFC}} E_{z} h}{A_{e}},
$$

where $Q$ is the electric charge, $A_{e}$ is the electrode area of MFC, $U$ is the voltage on MFC, and $h$ is a geometry constant of MFC, respectively.

In elastic deformation range of beam and MFC and with (1)-(3), the induced voltage is described as

$$
U_{m}=h_{\mathrm{MFC}} C_{\mathrm{MFC}} \varepsilon_{\mathrm{av}},
$$

where $U_{m}$ is the real-time open circuit voltage, $h_{\mathrm{MFC}}$ is an electromechanical constant of the MFC-beam coupled structure, $C_{\mathrm{MFC}}$ is the capacitance of MFC, and $\varepsilon_{\mathrm{av}}$ is the applied average strain of the energy harvesting MFC attached area, respectively.

Real-time energy on MFC can be calculated as

$$
e_{c}=\frac{1}{2} C_{\mathrm{MFC}} U_{m}^{2}
$$

where $e_{c}$ is the power output in one cycle.

For power outputted from energy harvesting MFC, the frequency of vibration is

$$
P_{s}=e_{c} f
$$

where $P_{s}$ and $f$ are the power output from MFC and frequency of vibration, respectively.

Then the power output from MFC can be calculated as

$$
P_{s}=\frac{1}{2} C_{\mathrm{MFC}} U_{m}^{2} f=\frac{1}{2} h_{\mathrm{MFC}}^{2} C_{\mathrm{MFC}}^{3} \varepsilon_{\mathrm{av}}^{2} f .
$$

Table 1 presents the parameters of MFC, cantilever beam, and whole vibrating structure. The first and second modes of cantilever beam without MFC attached are theoretically calculated as $8.42 \mathrm{~Hz}$ and $52.76 \mathrm{~Hz}$. The first and second modes of MFC-beam coupled structure are measured as $8.30 \mathrm{~Hz}$ and $51.99 \mathrm{~Hz}$ with finite element method (FEM).

2.2. Enhanced Energy Harvesting Circuit Design. Energy is harvested from the vibrating beam with the initial form of AC electric power. The general schematic diagram of the enhanced EH circuit is presented in Figure 2. Real-time voltage $U_{m}$ varies as the vibration amplitude changes. A fullbridge rectifier and stabilization capacitance are employed after MFC. DC voltage $U_{d}$ from the full-bridge rectified circuit and capacitance is still not fit for use because its amplitude is not stable and not fit for the requirement as 


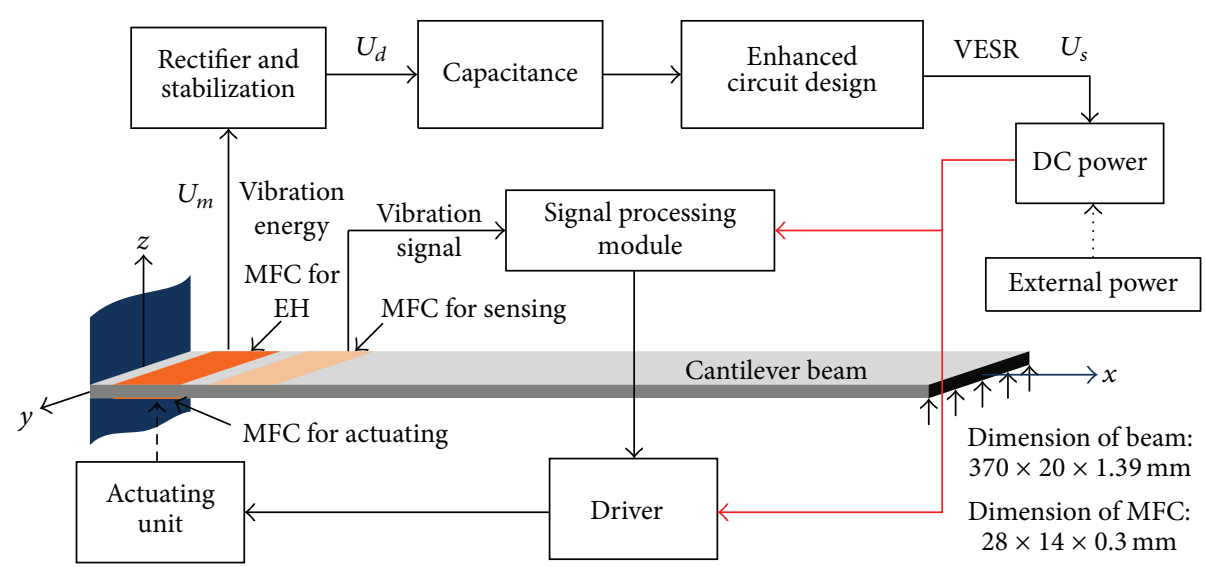

FIGURE 1: Block diagram of active vibration control with EH structure.

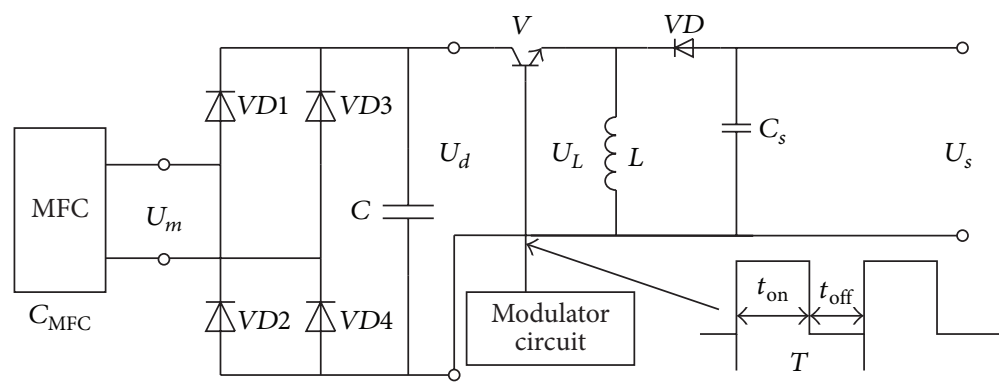

FIGURE 2: Schematic diagram of enhanced circuit design.

the vibration alters. Commercial DC-DC converter enlarges the input DC voltage range in some way and outputs stable DC voltage with a certain value. When structure vibrates in smaller or larger scale, the induced $U_{d}$ is lower or higher than the input range of the commercial DC-DC converter. Thus, a circuit with the function of adjusting DC voltage is necessary.

In Figure 2, VD1-VD4 is the full-bridge rectifier. $C$ is the filtering capacitance. $V$ is the switching transistor, which is controlled by a modulator circuit. The control signal is from the sensing MFC beside the energy harvesting MFC. When $t=t_{0}, V$ is on. Then power is supplied to inductance $L$. When $t=t_{1}, V$ is off. The current keeps on because the inductance restores energy. After one cycle, $V$ is on again. Then a new cycle starts. When the procedure is stable, it is known from inductance $L$ that

$$
\int_{0}^{T} U_{L} d t=0
$$

where $U_{L}$ is the real-time voltage on inductance $L$; $T$ is the cycle of modulator circuit. that

According to the stable condition in one cycle, it is known

$$
U_{d} t_{\text {on }}=U_{s} t_{\text {off }} \text {. }
$$

Then the output voltage can be calculated as

$$
U_{s}=\frac{t_{\mathrm{on}}}{T-t_{\mathrm{on}}} U_{d}=\frac{t_{\mathrm{on}}}{t_{\mathrm{off}}} U_{d}
$$

where $U_{s}$ is the average open circuit voltage of output, $t_{\text {on }}$ is the on-time of $V$, and $t_{\text {off }}$ is the off-time of a cycle.

It is known from (10) that when $t_{\text {on }}$ is larger than $t_{\text {off }}$, the voltage $U_{d}$ is boosted. When $t_{\mathrm{on}}$ is smaller than $t_{\text {off }}, U_{d}$ is bucked. This rate is controlled by SCM according to the sensing signals from sensing MFC. Based on the experiment reliability, $t_{\text {on }} / t_{\text {off }}$ is set in the range of $1 / 4 \sim 4$ in this work.

2.3. Schematic Explanation of Enhanced Circuit Design. The enhanced EH circuit converts the electric power from abandoned forms to accessible forms. It widens the energy harvesting range with wideband frequency of vibration and larger vibration amplitude range. This circuit enlarges the energy harvesting ability.

Figure 3 presents the function of the enhanced circuit design. When DC voltage from rectifier is off from the acceptance range of DC-DC converter, harvested energy is abandoned, as seen from the region above plane 1 and below plane 2 in Figure 3. With enhanced circuit design, the accessible range is enlarged. Plane 1 rises to plane $1^{\prime}$ and plane 2 drops to plane $2^{\prime}$. Then larger energy forms become accessible.

\section{Experiments}

In this work, experiments are primarily developed to obtain the electromechanical properties of the MFC-beam system. 


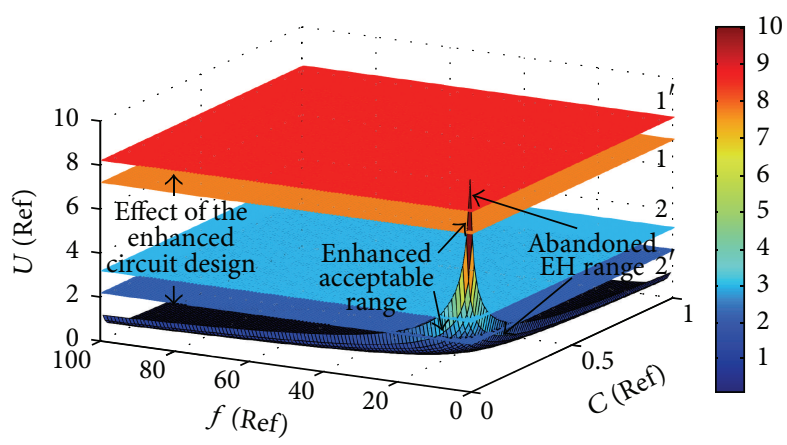

FIgURE 3: Function of enhanced circuit design.

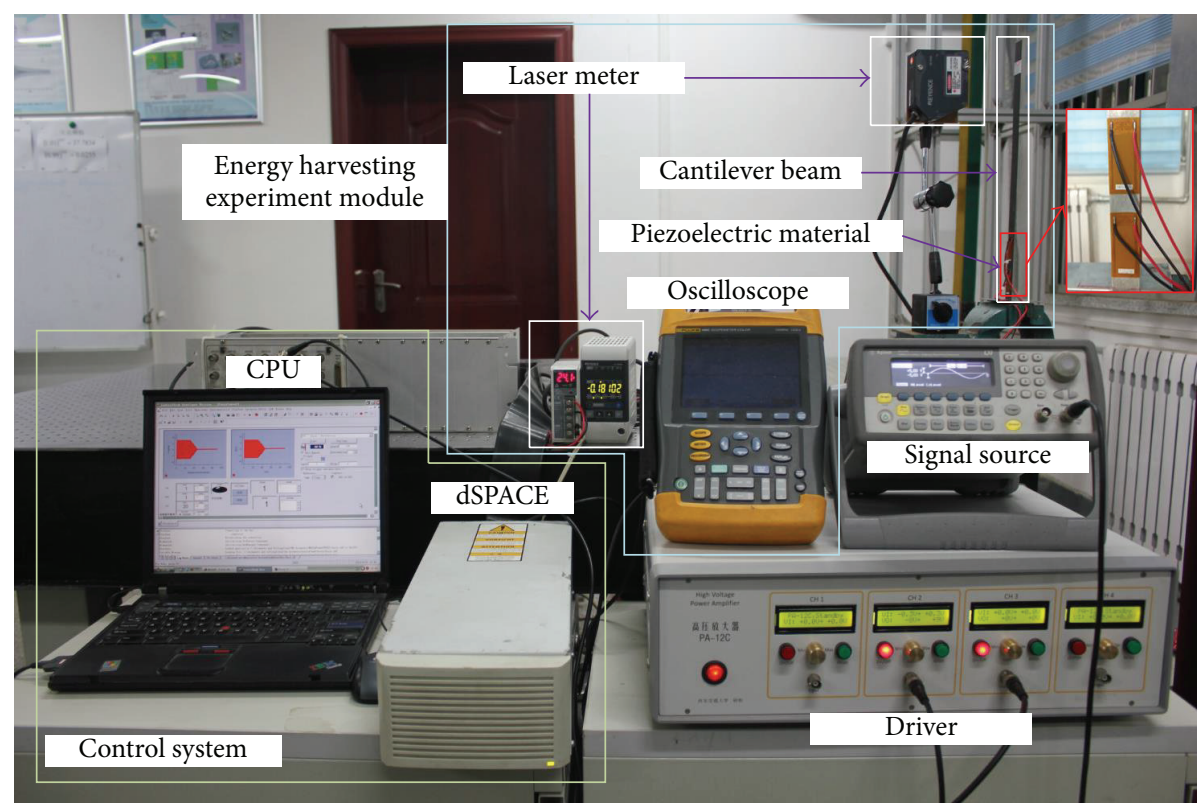

FIGURE 4: Outlook of active control system with EH.

The enhanced $\mathrm{EH}$ circuit is then tested to adjust the DC voltage value from MFC.

3.1. Experiment System. The energy harvesting system is a part of the active vibration control system. Figure 4 is the active vibration control system with $\mathrm{EH}$ work presented in this work. Dynamic signal is generated from the signal source, which is then sent to the piezoelectric material driver. The driver outputs the driving voltage to the actuating MFC and makes the structure vibrate. The vibration amplitude and frequency are tested by the laser sensing system. The $\mathrm{EH}$ MFC captures the vibration energy and converts it to AC electric voltage form. The enhanced $\mathrm{EH}$ circuit converts the electric power form and adjusts the voltage value mentioned in Section 2.2. The control signal is from the sensing MFC.

\subsection{Electromechanical Properties of EH Structure. Figure 5} reflects the electromechanical properties of the $\mathrm{EH}$ structure. Figure 5(a) is the relationship between the displacement on laser sensing point and the applied average strain of the $\mathrm{EH}$ region. It is clear from Figure 5(a) that in $0-3.2 \mathrm{~mm}$, applied average strain of the $\mathrm{EH}$ region is proportional to the displacement of the sensing point. Figure 5(b) is the charge output-strain curve from MFC.

3.3. Measurement of Electric Output. A real-time waveform of MFC output and displacement is given in Figure 6. Since the oscilloscope is straightly connected with $\mathrm{EH}$ MFC to observe the output waveform, the real open circuit voltage generated from EH MFC (seen from Figure 6) is not the same as the data measured by oscilloscope. The real voltage value is calculated according to the impedance rate of oscilloscope and EH MFC at the vibration frequency. The ratio of the measured value and the real value (at $8.3 \mathrm{~Hz}$ ) is calculated as

$$
\gamma=\frac{U_{m}}{U_{\text {mea }}}=\left|\frac{Z_{\mathrm{osc}}+Z_{\mathrm{MFC}}}{Z_{\mathrm{osc}}}\right|=7.143 \text {, }
$$

where $U_{\text {mea }}$ is the measured voltage value of oscilloscope, $Z_{\text {osc }}$ is the impedance of oscilloscope at $8.3 \mathrm{~Hz}$, and $Z_{\mathrm{MFC}}$ is the impedance of MFC at $8.3 \mathrm{~Hz}$, respectively. 


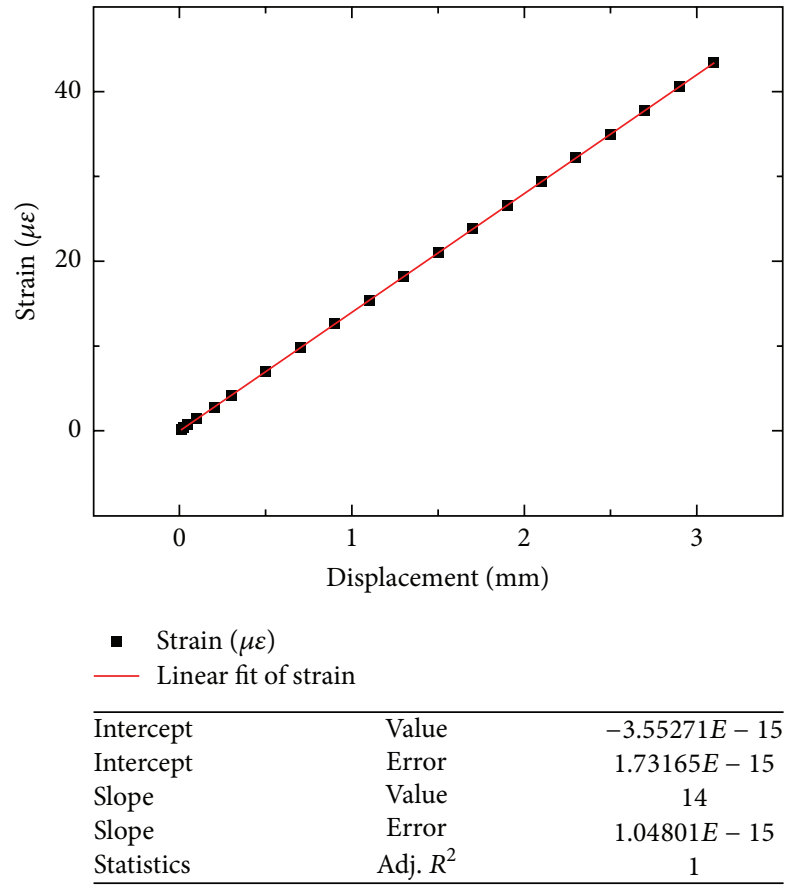

(a)

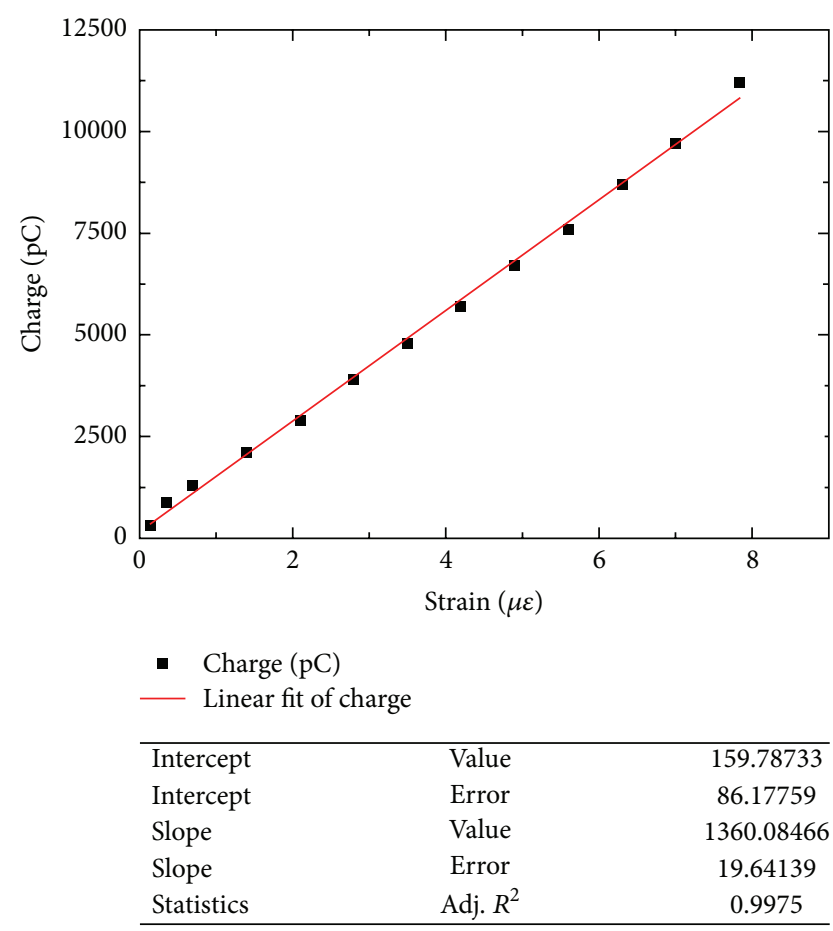

(b)

FIGURE 5: Electromechanical properties of EH structure: (a) the relationship between sensing point displacement of structure and the average strain of the $\mathrm{EH}$ region, (b) the relationship between the average strain of the $\mathrm{EH}$ region and the charge output.

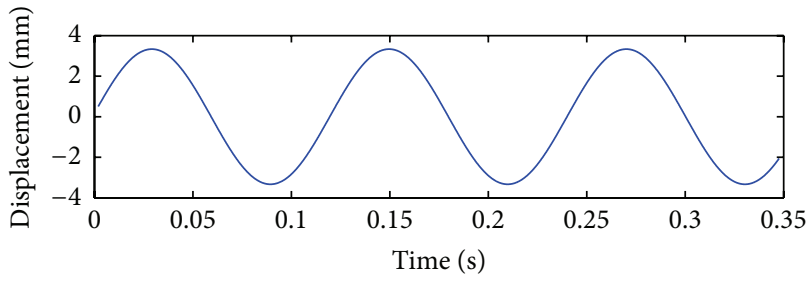

— Displacement

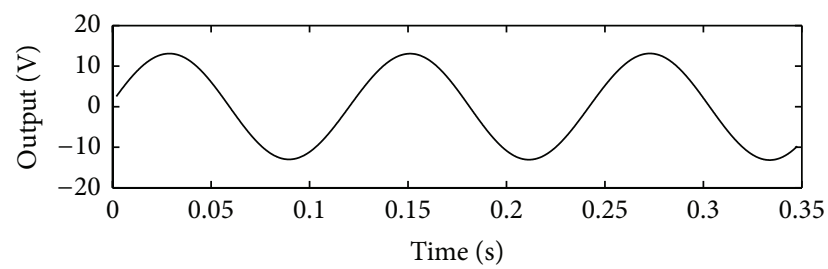

- Output

FIGURE 6: MFC real-time waveform of displacement and output.

Figure 7 presents the real-time voltage output and its rectified waveform displayed on oscilloscope when the sensing vibrating amplitude is $3.2 \mathrm{~mm}$.

As piezoelectric materials show capacitive property, the real-time voltage value on MFC is also calculated as

$$
U_{m}=\frac{Q_{c}}{C_{\mathrm{MFC}}}
$$

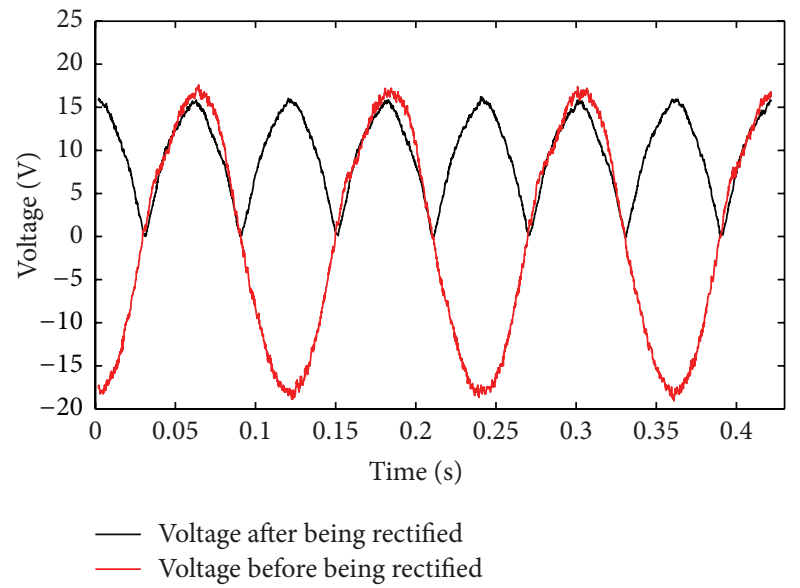

Figure 7: Displayed voltage output before and after rectification (sensing point vibrating amplitude: $3.2 \mathrm{~mm}$ at $8.30 \mathrm{~Hz}$ ).

where $Q_{c}$ is the charge value on the EH MFC. To smooth the DC waveform and restore energy, a capacitance $C_{s}$ is connected behind the rectifier. $C_{s}=10 \mu \mathrm{F}$ is adopted in this experiment. DC voltage increases as approximately $2.2 \mathrm{~V} / \mathrm{s}$ on capacitance $C_{s}$ as the $8.30 \mathrm{~Hz}$ vibrating amplitude is $3.2 \mathrm{~mm}$ on sensing edge.

Figure 8 is the DC waveform from the enhanced circuit design output at different $t_{\text {on }} / t_{\text {off }}$ conditions. The output DC voltage is able to be increased or decreased by raising or decreasing the duty ratio $\left(t_{\text {on }} / t_{\text {off }}\right)$ of the control signal. When 


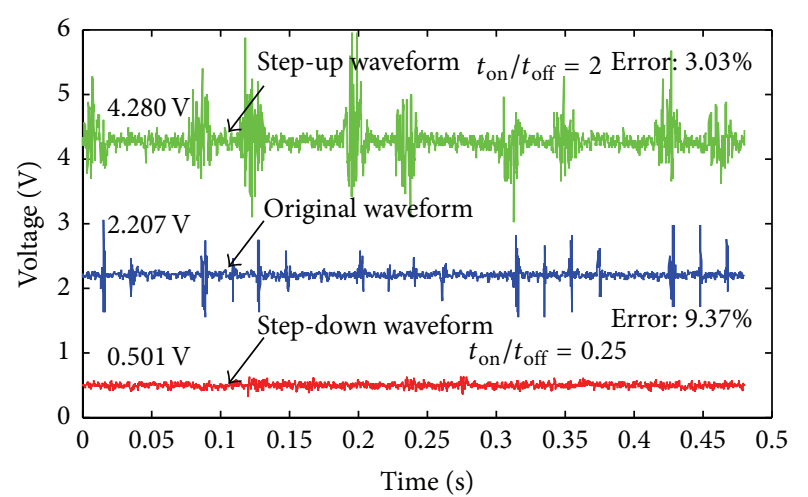

FIGURE 8: Comparison between original DC waveform and waveforms output from enhanced circuit.

the vibration amplitude is lower than the floor level of DCDC converter, the enhanced circuit increases the voltage amplitude into the input available range. When voltage is higher than the upper level of DC-DC converter, it decreases the DC voltage as the same way. Burrs can be smoothed by capacitance.

\section{Energy Harvesting Ability Analysis}

The vibration energy self-supply ratio (VESR) is defined to describe the energy harvesting ability. In this discussion, the load value is defined as $2 \mathrm{~mW}$. When harvested energy is surplus, the extra part will be restored (VESR > 1). The vibrating energy may just be sufficient for supplying the load (VESR $=1)$. When vibration energy harvesting quantity is not sufficient for load, VESR is necessary to explain the EH efficiency $(\mathrm{VESR}<1)$.

The VESR is defined as

$$
\chi=\frac{P_{s}}{P} \times 100 \%
$$

where $\chi$ is VESR of EH power supply and $P$ is the power requirement of the signal processing circuit, respectively.

When the harvested energy is surplus, the extra energy is restored on EH capacitance $(\chi>1)$. When $\chi=1$, the $\mathrm{EH}$ energy is just sufficient for load. The adjusted energy becomes a part of the power for load when $\chi<1$. In this analysis, the two resonance frequencies mentioned in Section 2.1 are used to discuss the energy harvesting effect. Since surplus energy is restored in $\mathrm{EH}$ capacitance, the analysis just focuses on the situation of $\chi \leq 1$. Figure 9 reflects the energy harvesting effect when $\chi \leq 1$. With the enhanced circuit design, the accessible range of $8.3 \mathrm{~Hz}$ vibration has expanded from $167.48 \mu \varepsilon \sim 334.95 \mu \varepsilon$ to $41.86 \mu \varepsilon \sim 1339 \mu \varepsilon$. The accessible range of $51.99 \mathrm{~Hz}$ vibration has expanded from $67.2 \mu \varepsilon \sim 134.4 \mu \varepsilon$ to $16.83 \mu \varepsilon \sim 537.6 \mu \varepsilon$.

Figure 10 reflects the effect of the enhanced $\mathrm{EH}$ circuit in different vibration frequencies. $\mathrm{EH}$ ability is enlarged with the enhanced circuit design: area between the accessible upper limit and the accessible lower limit is enlarged in different vibration frequencies.

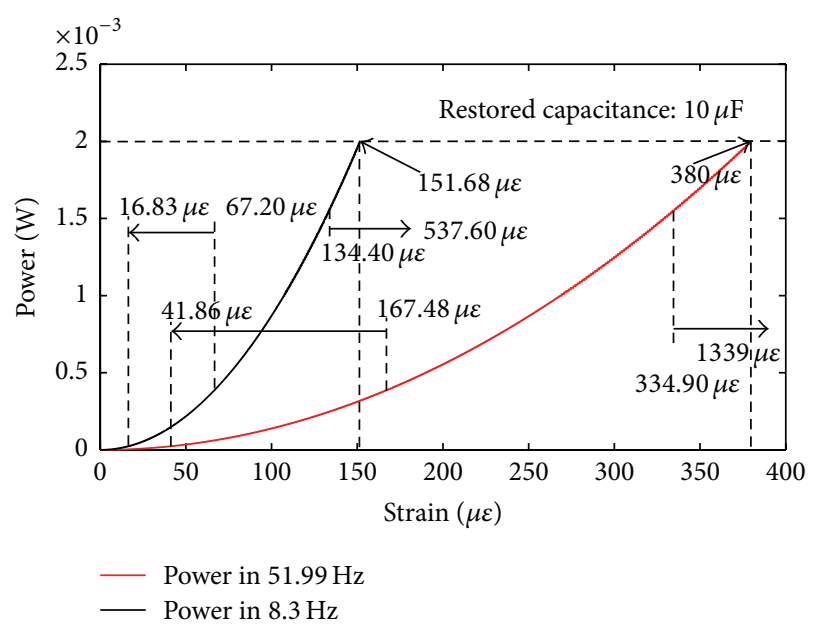

FIGURE 9: Energy harvesting effect of the EH MFC with the enhanced circuit.

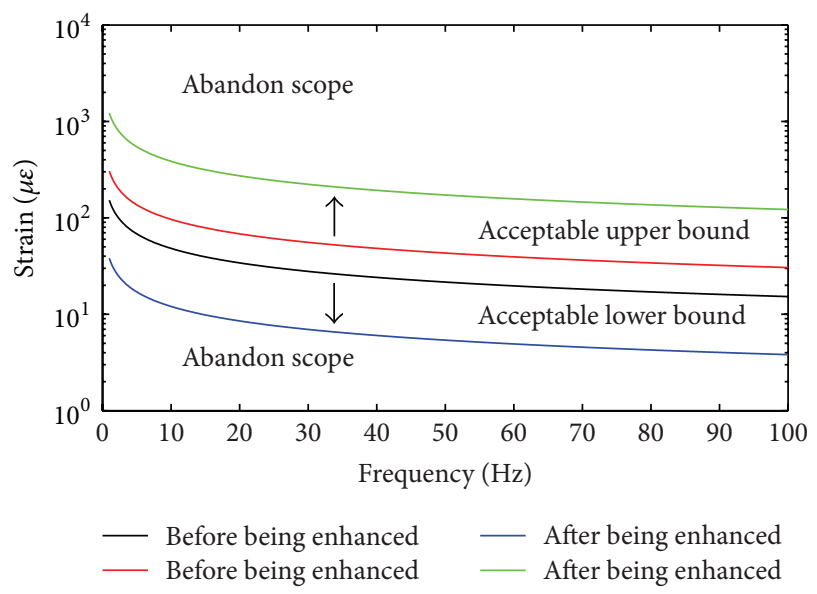

Figure 10: Effect of the enhanced circuit design.

Figure 11 reflects this relationship between the power output and the impedance ratio of EH system and the load when the vibration is at 2 resonant frequencies. It is seen from Figure 11 that when the load impedance value is the same as the EH system, the energy output reaches the maximum value.

\section{Conclusion}

In this work, an enhanced vibration energy harvesting system with MFC is presented. The electromechanical coupling properties and function of enhanced $\mathrm{EH}$ circuit have been analyzed. Experiments are developed to test the electric output of the EH system. The experimental result proves that the enhanced circuit design is able to step up and step down the DC voltage in wide frequency range. This work helps harvest more vibration energy. The relationship between power and load impedance is provided for optimizing the circuit impedance as load varies. With the experimental EH method presented in this work, the piezoelectric-based energy harvesting ability is increased. 


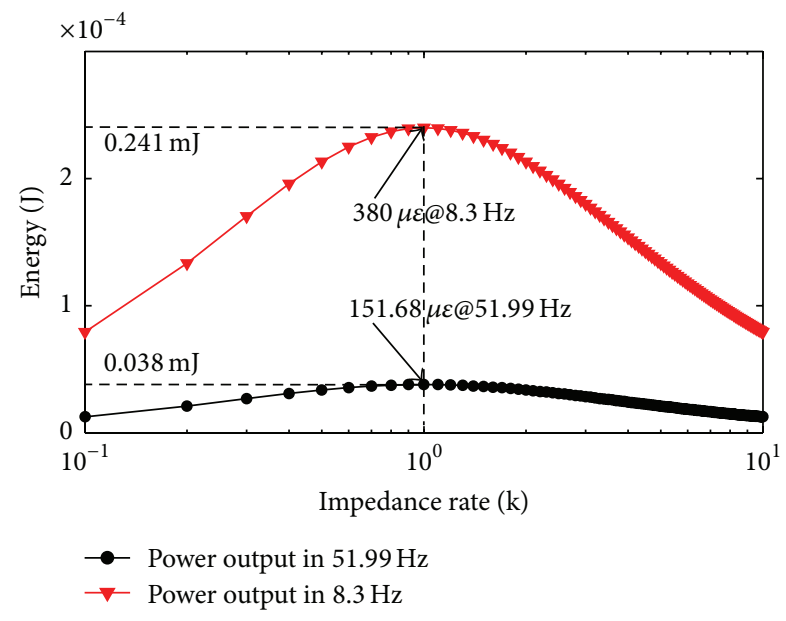

FIGURE 11: Impedance rate-power output curve in two vibration frequencies.

\section{Conflict of Interests}

The authors declare that there is no conflict of interests regarding the publication of this paper.

\section{Acknowledgment}

This work is supported by the National Natural Science Foundation of China (Grant nos. 11172229 and 11321062).

\section{References}

[1] V. Mudupu, M. B. Trabia, W. Yim, and P. Weinacht, "Design and validation of a fuzzy logic controller for a smart projectile fin with a piezoelectric macro-fiber composite bimorph actuator," Smart Materials and Structures, vol. 17, no. 3, Article ID 035034, 2008.

[2] J. Schröck, T. Meurer, and A. Kugi, "Control of a flexible beam actuated by macro-fiber composite patches: I. Modeling and feedforward trajectory control," Smart Materials and Structures, vol. 20, no. 1, Article ID 015015, 2011.

[3] H. A. Sodano, G. Park, and D. J. Inman, "An investigation into the performance of macro-fiber composites for sensing and structural vibration applications," Mechanical Systems and Signal Processing, vol. 18, no. 3, pp. 683-697, 2004.

[4] J. Schrock, T. Meurer, and A. Kugi, "Control of a flexible beam actuated by macro-fiber composite patches: II. Hysteresis and creep compensation, experimental results," Smart Materials and Structures, vol. 20, Article ID 015016, 2011.

[5] B. Yan, X. Zhang, and H. Niu, "Design and test of a novel isolator with negative resistance electromagnetic shunt damping," Smart Materials and Structures, vol. 21, no. 3, Article ID 035003, 2012.

[6] H. Painter and J. Flynn, "Current and future wet-mate connector technology developments for scientific E scabbed observatory application," in Proceedings of the MTS/IEEE OCEANS, pp. 881-886, Boston, Mass, USA, September 2006.

[7] T. Kojiya, F. Sato, and H. Matsuki, "Construction of noncontacting power feeding system to underwater vehicle utilizing electromagnetic induction," in Proceedings of the Oceans 2005Europe, vol. 1-2, pp. 709-712, 2005.

[8] X. Zhang, H. Niu, and B. Yan, "A novel multimode negative inductance negative resistance shunted electromagnetic damping and its application on a cantilever plate," Journal of Sound and Vibration, vol. 331, no. 10, pp. 2257-2271, 2012.

[9] A. Erturk and D. J. Inman, "An experimentally validated bimorph cantilever model for piezoelectric energy harvesting from base excitations," Smart Materials and Structures, vol. 18, no. 2, Article ID 025009, 2009.

[10] K. A. Cook-Chennault, N. Thambi, and A. M. Sastry, "Powering MEMS portable devices-a review of non-regenerative and regenerative power supply systems with special emphasis on piezoelectric energy harvesting systems," Smart Materials and Structures, vol. 17, no. 4, Article ID 043001, 2008.

[11] M. Lallart and D. Guyomar, "An optimized self-powered switching circuit for non-linear energy harvesting with low voltage output," Smart Materials and Structures, vol. 17, no. 3, Article ID 035030, 2008.

[12] J.-Q. Liu, H.-B. Fang, Z.-Y. Xu et al., "A MEMS-based piezoelectric power generator array for vibration energy harvesting," Microelectronics Journal, vol. 39, no. 5, pp. 802-806, 2008.

[13] D. Shen, J.-H. Park, J. H. Noh et al., "Micromachined PZT cantilever based on SOI structure for low frequency vibration energy harvesting," Sensors and Actuators, A: Physical, vol. 154, no. 1, pp. 103-108, 2009.

[14] H.-B. Fang, J.-Q. Liu, Z.-Y. Xu et al., "Fabrication and performance of MEMS-based piezoelectric power generator for vibration energy harvesting," Microelectronics Journal, vol. 37, no. 11, pp. 1280-1284, 2006.

[15] M. Ferrari, V. Ferrari, M. Guizzetti, B. Andò, S. Baglio, and C. Trigona, "Improved energy harvesting from wideband vibrations by nonlinear piezoelectric converters," Procedia Chemistry, vol. 1, no. 1, pp. 1203-1206, 2009.

[16] F. Cottone, L. Gammaitoni, H. Vocca, M. Ferrari, and V. Ferrari, "Piezoelectric buckled beams for random vibration energy harvesting," Smart Materials and Structures, vol. 21, no. 3, Article ID 035021, 2012.

[17] B. Yang, C. Lee, W. Xiang et al., "Electromagnetic energy harvesting from vibrations of multiple frequencies," Journal of Micromechanics and Microengineering, vol. 19, no. 3, Article ID 035001, 2009.

[18] L. Gu and C. Livermore, "Impact-driven, frequency up-converting coupled vibration energy harvesting device for low frequency operation," Smart Materials and Structures, vol. 20, no. 4, Article ID 045004, 2011.

[19] A. F. Arrieta, P. Hagedorn, A. Erturk, and D. J. Inman, "A piezoelectric bistable plate for nonlinear broadband energy harvesting," Applied Physics Letters, vol. 97, no. 10, Article ID 104102, 2010.

[20] S. C. Stanton, C. C. McGehee, and B. P. Mann, "Nonlinear dynamics for broadband energy harvesting: investigation of a bistable piezoelectric inertial generator," Physica D: Nonlinear Phenomena, vol. 239, no. 10, pp. 640-653, 2010.

[21] M. Ferrari, V. Ferrari, M. Guizzetti, B. Andò, S. Baglio, and C. Trigona, "Improved energy harvesting from wideband vibrations by nonlinear piezoelectric converters," Sensors and Actuators, A: Physical, vol. 162, no. 2, pp. 425-431, 2010.

[22] R. Torah, P. Glynne-Jones, M. Tudor, T. O’Donnell, S. Roy, and S. Beeby, "Self-powered autonomous wireless sensor node using vibration energy harvesting," Measurement Science and Technology, vol. 19, no. 12, Article ID 125202, 2008. 

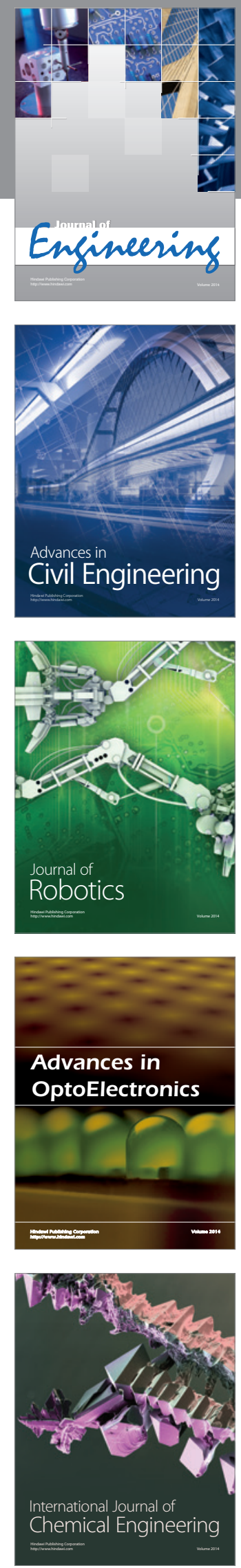

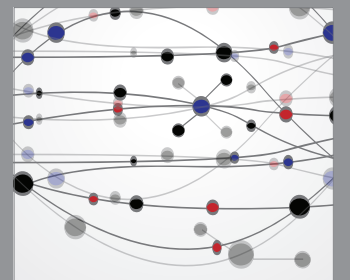

The Scientific World Journal
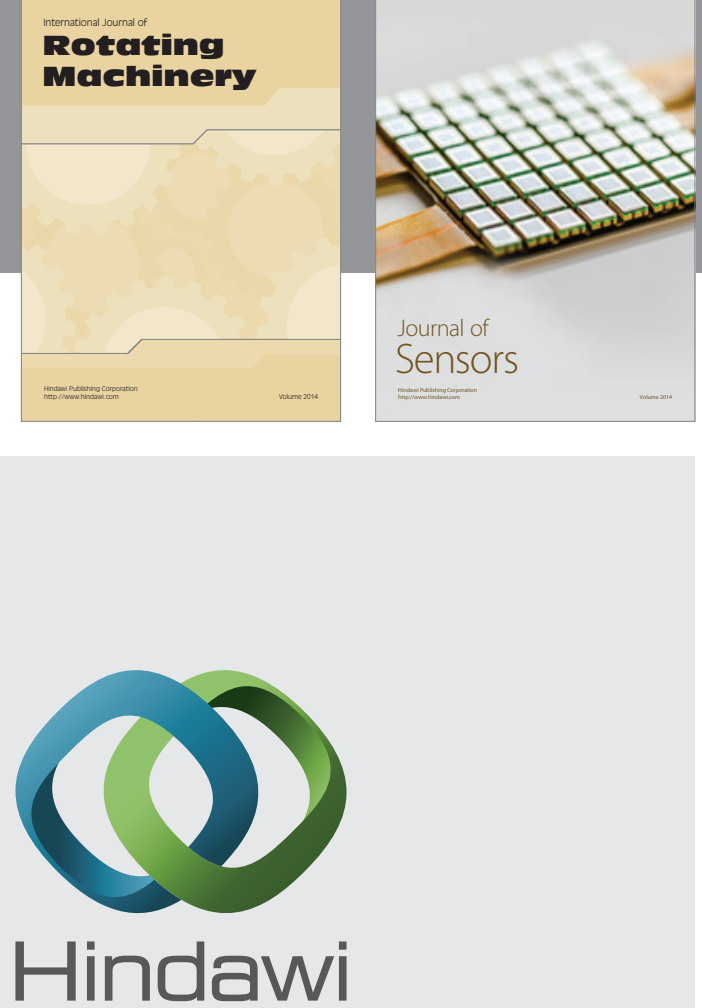

Submit your manuscripts at http://www.hindawi.com
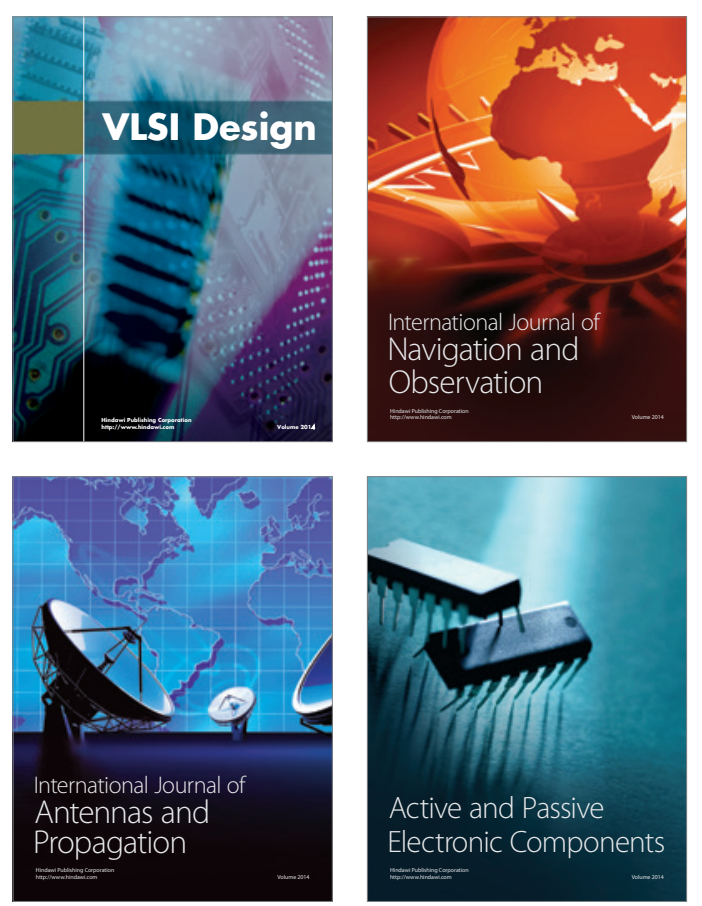
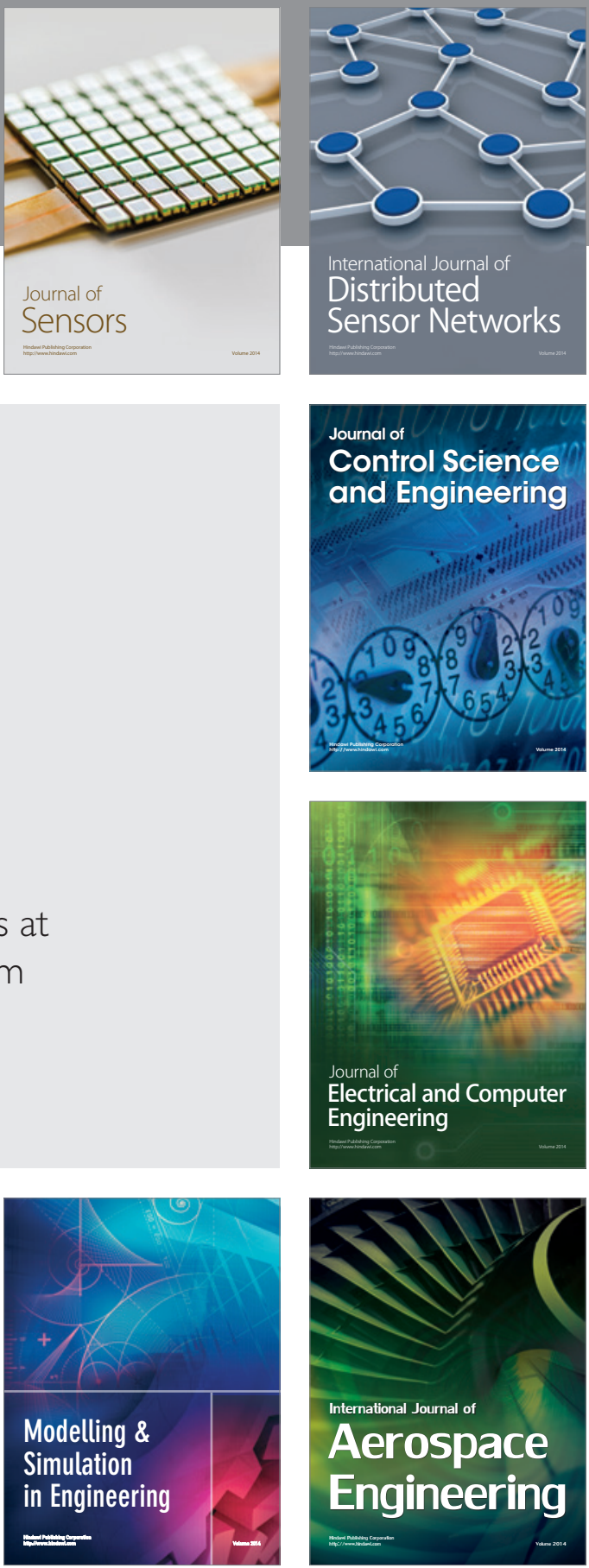

Journal of

Control Science

and Engineering
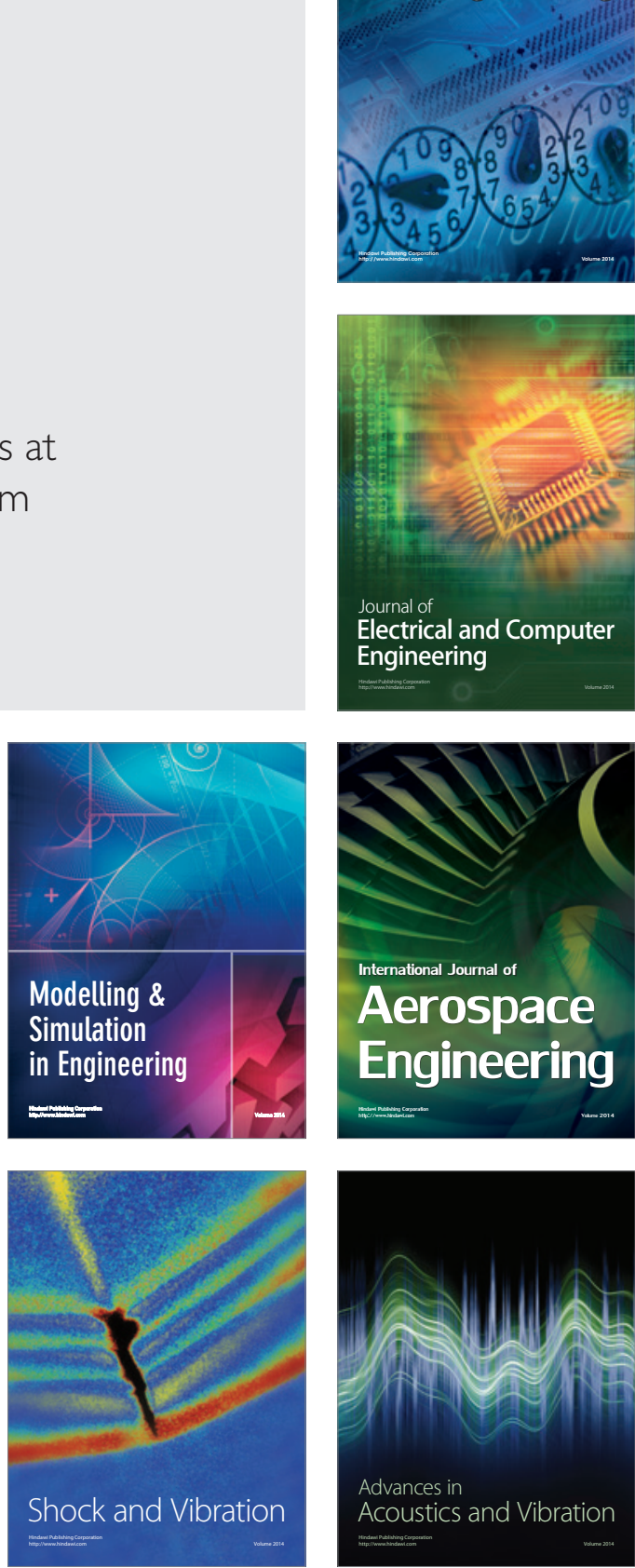